1 TITLE: Real-Time Non-Intrusive Assessment of Viewing Distance during

\title{
Computer Use
}

3 Authors:

Marc Argilés, $\mathrm{MSc}^{\wedge}$

4

Genís Cardona, $\mathrm{PhD}^{\wedge}$

5

Elisabet Pérez-Cabré, PhD ^

6

Ramon Pérez-Magrané, PhD \&

7

Bernardo Morcego, $\mathrm{PhD}^{\text {\& }}$

8

Joan Gispets, $\mathrm{PhD} \wedge$

9

^Department of Optics and Optometry, School of Optics and Optometry of Terrassa, Universitat Politècnica de Catalunya $\cdot$ BarcelonaTech, Terrassa, Spain

\& Department of Automatic Control, Terrassa School of Engineering, Universitat Politècnica de Catalunya · BarcelonaTech, Terrassa, Spain

N. Tables: $\mathbf{2}$

N. Figures: $\mathbf{5}$

N. Words: 4155

Genís Cardona (gcardona@oo.upc.edu)

c/ Violinista Vellsolà, 37

E08222 Terrassa, Catalonia, Spain

+34937398774 
Purpose: to develop and test the sensitivity of an ultrasound-based sensor to assess the viewing distance of visual display terminals operators in real time conditions.

Methods: A modified ultrasound sensor was attached to a computer display to assess viewing distance in real time. Sensor functionality was tested on a sample of 20 healthy participants while they conducted four 10-minute randomly presented typical computer tasks (a matchthree puzzle game, a video documentary, a task requiring participants to complete a series of sentences and a predefined internet search).

Results: The ultrasound sensor offered good measurement repeatability. Game, text completion and web search tasks were conducted at shorter viewing distances $(54.4 \mathrm{~cm}$ [Cl95\% 51.3 to $57.5 \mathrm{~cm}$ ], $54.5 \mathrm{~cm}$ [Cl95\% 51.1 to $58.0 \mathrm{~cm}$ ] and $54.5 \mathrm{~cm}$ [Cl95\% 51.4 to $57.7 \mathrm{~cm}$ ], respectively) than the video task $(62.3 \mathrm{~cm}$ [CI95\% 58.9 to $65.7 \mathrm{~cm}])$. Statistically significant differences were found between the video task and the other three tasks (all $p<0.05$ ). Range of viewing distances (from 22 to $27 \mathrm{~cm}$ ) was similar for all tasks ( $F=0.996 ; p=0.413$ ). intrusive ultrasonic device disclosed a task-dependent pattern.

\section{KEY WORDS}


Recent decades have witnessed a constant increase in the number of computers at home and at the workplace, with a reported $78.9 \%$ of US households owning a computer in $2012^{1}$ and an estimated 2 billion computers worldwide in 2014. Computers and visual display terminals (VDT), ranging from smartphones to desktop computers and TVs, are ubiquitous in developed countries, and serve as a platform for internet access, gaming, communication, entertainment and work.

Hultgren and Knave ${ }^{2}$ first documented visual fatigue in computer workers in 1974, and the term Computer Vision Syndrome (CVS) was later introduced to describe a constellation of body, ocular and visual symptoms affecting this population. These manifestations, which are particularly well documented in desktop computer users, may include headache, tired eyes, irritation, dry eyes, blurred vision at near or distance (after prolonged near vision work) and double vision. ${ }^{3-7}$ Symptoms are influenced, among other factors, by actual VDT exposure time, type of screen, background luminance, glare sources and distance from the screen..$^{8-11}$

In particular, viewing distance in computer users is the focus of interest of many studies on CVS and ergonomics, even though there is a large discrepancy regarding the actual definition of correct viewing distance, with a range from 30 to $100 \mathrm{~cm}$. Indeed, short viewing distances have been related to an increased visual strain, ${ }^{8,12}$ with users reporting fewer symptoms of visual fatigue at $100 \mathrm{~cm}$ than at $50 \mathrm{~cm}$ when font size is adjusted to provide the same visual angle, and regardless of individual dark-focus point (that is, resting level of accommodation and vergence, which is about $67 \mathrm{~cm}) .{ }^{13}$ In this regard, it is not surprising that the various international standards also recommend different viewing distances. ${ }^{14}$ For instance, the EN ISO 9241- $5^{15}$ suggests a viewing distance of $60 \mathrm{~cm} \pm 15 \mathrm{~cm}$; the US MIL STD 1472-C $\mathrm{C}^{16}$ states an average distance of $40 \mathrm{~cm}$ for continuous viewing and a minimum distance of $25 \mathrm{~cm}$ for intermittently viewed displays; finally the DIN $66234^{17}$ proposes a range between 45 and 60 
$\mathrm{cm}$, but recommends $50 \mathrm{~cm}$ when frequent refixations are necessary between keyboard or source documents and VDT. ${ }^{18}$

In addition, viewing distance has been found to depend on such factors as size and resolution of visual stimuli, type of $\operatorname{task}^{19}$ and screen/text color combination. Thus, for example, given the recommendation of adopting a $3 x$ acuity reserve, ${ }^{20}$ a shorter viewing distance is associated with prolonged viewing of texts in fonts of small size. Similarly, white text on blue background was found to result in a greater viewing distance $(60.3 \mathrm{~cm})$ than same size red text on green background $(47.4 \mathrm{~cm})$, with observers viewing text at the commonly employed black on white combination at an average distance of $56.9 \mathrm{~cm} .{ }^{12}$

It is also relevant to mention that myopia onset and progression, defined as an increase in myopia of more than -0.25 dioptres (D) and up to $-1.00 \mathrm{D}$ per year, ${ }^{21,22}$ have been related to both viewing distance for near work and the amount of time spent conducting near work tasks. ${ }^{23-25}$ In view of the global socio-economic impact of myopia, ${ }^{26}$ it may be of interest to accurately monitor viewing distance in order to gain a better understanding of the contribution of distance as a possible risk factor of and to develop strategies to prevent myopia onset and progression.

Considering the documented association between viewing distance, visual fatigue, CVS and myopia onset and progression, the current lack of an affordable, non-intrusive means of determining viewing distance in real time is unexpected. In this regard, only two previous attempts at measuring viewing distance in real time were uncovered in our literature review. On the one hand, Eastwood-Sutherland and Gale developed an infra-red system consisting of a video camera and infra-red LED markers attached to the forehead or the back of the head of VDT operators. ${ }^{19}$ With this instrument, the authors were able to document changes in viewing distance with a temporal resolution of $7 \mathrm{~Hz}$, noting a link between the type of activity being 
97 conducted and distance, with internet browsing resulting in shorter viewing distances than

98 writing a text. On the other hand, Piccoli and co-workers designed an ultrasound emitter (at 40

$99 \mathrm{KHz}$ ), coupled with a receiving sensor placed on the operator's forehead ${ }^{27}$ with which the 100 authors reported an accuracy of $\pm 0.5 \mathrm{~cm}$ and a maximum temporal resolution of $10 \mathrm{~Hz}$ when 101 measuring viewing distance on a sample of VDT users.

102 It may be noted that both approaches require sophisticated equipment and controlled 103 experimental settings (placing a sensor or marker on the forehead of the VDT operator) that 104 prevent their simple implementation in a real life environment such as an office or school. It was therefore the main goal of the present study to develop an automated sensor, based on ultrasound technology, easily attachable to any VDT and adaptable to any working environment, as well as the accompanying software, to measure viewing distance of computer users in real time conditions. To test whether our sensor was able to detect small changes in viewing distance, measurements were conducted on a sample of participants performing four typical computer tasks. As previous investigators have described an influence of the type of

111 task on viewing distance, ${ }^{19}$ this research question was also explored. 
115 A group of 20 volunteers was recruited to participate in the present study, which took place in

116 the facilities of a high school in the city of Lleida (Spain) between April and May 2014.

117 Participants were selected at random from those attending a word processor workshop in the computer classroom. Inclusion criteria were age between 14 and 25 years (inclusive), spherical and corrected monocular and binocular visual acuity at distance and near equal or better than $0.0 \log M A R$. Patients presenting any eye disease, dry eye, binocular vision abnormalities, amblyopia or anisometropia $>1.00 \mathrm{D}$ were excluded from the study, as were those showing low cooperation with the study protocol, defined as the inability to comply with the given instructions, mainly to conduct each task during the predetermined 10-minute interval and in silence. Both spectacle and contact lens wearers were included in the study.

All participants provided written informed consent after the nature of the study was explained to them, although details regarding the specific aim of the investigation (distance evaluation) were not revealed until the completion of the study. Parental consent was obtained for those participants who were underage. The study was conducted in accordance with the Declaration of Helsinki tenets of 1975 (as revised in Tokyo in 2004) and received the approval of the Ethics Review Board of the Hospital Universitari Mútua de Terrassa. composed by members of the Departments of Automatic Control and Optics and Optometry of 

components. Essentially, the hardware measurement subsystem is a modified ultrasonic range finder (SFR02, Devantech Ltd. [Robot Electronics], Norfolk, England), which is a small distance sensor, typically used in robotic applications, with a range from $0.15 \mathrm{~m}$ to $6 \mathrm{~m}$ within its detection field (Figure 1). It must be noted that detection sensitivity decreases with distance from the sensor, although it remains high within the range of distances that are relevant for the purpose of the present study (see Figure 1). This sensor uses two standard communication interfaces (I2C and serial) which are not very common in personal computers. Therefore, an adapter was employed to convert the I2C protocol to USB and to supply power to the device. The two boards with the main sensor and the I2C to USB adapter are small enough to fit into the casing of a standard webcam, as shown in Figure 2, which is convenient to mount the whole device on a computer display.

The software processing subsystem of the distance sensor is programmed in Java (Oracle systems. Although the software can work both remotely and on-site, in the first version of the sensor only the on-site functionality was considered. Three main operations are included in the software: sensor set-up, distance acquisition and display and data recording. The set-up process is based on the software provided by the manufacturer and is almost plug-and-play. The automatic calibration of the sensor is executed as soon as the application is started, whereupon, according to the manufacturer, the sensor does not require any further calibration, relying instead on an automatic tuning algorithm working continuously in the background to ensure correct measurements. Distance is measured every 7 seconds by default, although this time interval may be adjusted as necessary, with a maximum temporal resolution of $100 \mathrm{~ms}$. All measurements are stored in a file in which local date is recorded, as well as the local time associated with each measurement. Sensor resolution is $1 \mathrm{~cm}$. 
161 The software may be configured to present a red or green notification at the right lower edge

162 of the screen when observers are beyond or within the recommended viewing distance, respectively. This distance may be adjusted at any value, for example at $40 \mathrm{~cm}$.

Procedure

Notwithstanding the automatic continuous calibration described by the manufacturer, prior to monitoring viewing distance in human participants in the classroom, a preliminary study was conducted to investigate whether the sensor offered repeatable recordings at various controlled testing distances. In this occasion, an object was placed at exactly $20,40,60,80$ and $100 \mathrm{~cm}$ from the sensor and 10 consecutive measurements were obtained of each distance over the course of approximately one minute. The mean and variance of the readings obtained at each distance were determined. The range of tested distances (20 to $100 \mathrm{~cm}$ ) was selected as this is the range that may be considered useful for the purpose of monitoring viewing distance of VDT operators.

Participants were recruited following a complete optometric examination in accordance with the inclusion and exclusion criteria. At the beginning of each session, participants were instructed and assisted to sit in front of the computer at a distance of $60 \mathrm{~cm}$ and to adjust the height of their chairs and configuration of armrests to ensure comfort and to align the top of the screen at eye level. The inclination angle of the screen was of 100 degrees from the horizontal plane of the computer desk. Computer screens were 20 inch liquid crystal displays (TFT-LCD) set to a resolution of 1280 per 1024 pixels, 32 bit colour configuration and $75 \mathrm{~Hz}$ refresh rate. Measurements were simultaneously conducted on three computers adjusted to exactly the same configuration. 
184 Four different typical computer tasks were presented to the participants in a random order.

185 Tasks consisted in a popular match-three puzzle game, a video documentary, a task requiring participants to complete a series of sentences and a predefined internet search. Display luminance was approximately equivalent for all tasks (about $210 \mathrm{~cd} / \mathrm{m}^{2}$, as measured with a light meter [Gossen Mavolux 5032; Gossen Foto- und ichtmesstechnik GmbH, Nürnberg, Germany] with the luminance attachment). When necessary, tasks were completed with the aid of a keyboard and mouse combination. Task duration was set at 10 minutes and distance from the screen was readjusted at $60 \mathrm{~cm}$ between tasks. As noted above, for each 10-minute task and participant, distance measurements (in $\mathrm{cm}$ units) were recorded every 7 seconds. The average distance for each task and participant was then calculated, as well as the corresponding range of viewing distances, defined by the difference between maximum and minimum viewing distances (in $\mathrm{cm}$ units) recorded during that particular 10-minute interval.

Room illuminance was provided by indirect lighting in order to avoid glare sources, and was maintained at about $500 \mathrm{~lx}$. Room temperature and humidity were constantly monitored throughout the experimental sessions and remained approximately constant at about $22^{\circ} \mathrm{C}$ and between 53 and 58\%, respectively. Sessions took place during the mornings of consecutive days. All measurements were conducted while participants used their habitual visual correction.

202

Data analysis

Statistical analysis of the data was performed with the SPSS software 19.0 (IBM Corp., NY, US) for Windows. All data were examined for normality using the Kolmogorov-Smirnov test, which revealed normal distributions for all variables. Therefore, descriptive statistics present results regarding distance and range as the averages of all participants for each task and the 
corresponding standard deviation (SD). An analysis of variance test (ANOVA) was subsequently

209 employed to explore the statistical significance of the differences between the four tasks in average viewing distances and average range of distances and, when statistical significance was found, a post-hoc Bonferroni test was used for pair-wise analyses of the differences between tasks in these parameters. Possible associations between the average viewing distances of the different tasks were investigated with a Pearson's test of correlation. A p-

214 value of 0.05 or less was considered to denote statistical significance throughout the study. 
217 Study sample demographics

218 Twenty young subjects participated in the study (11 were female), with an age of $17.07 \pm 3.14$

219 years (mean \pm SD). Ten participants had myopia, 6 hyperopia and 4 were emmetropes, with an 220 average spherical refractive error of the study sample of $-1.23 \mathrm{D}( \pm 0.72 \mathrm{D})$.

221

Sensor operability

Sensor repeatability at the controlled distances of $20 \mathrm{~cm}, 40 \mathrm{~cm}, 60 \mathrm{~cm}, 80 \mathrm{~cm}$ and $100 \mathrm{~cm}$ was good. Maximum and minimum variance values of $0.16 \mathrm{~cm}$ at $100 \mathrm{~cm}$ and $0.09 \mathrm{~cm}$ at $20 \mathrm{~cm}$, respectively, were obtained. The average of the 10 measurements was centered at the corresponding distance under evaluation.

Viewing distance values for each task are summarized in Table 1. Results are presented as average distance (Figure $\mathbf{3}$ ) and range of viewing distances (difference between maximum and minimum viewing distance) (Figure 4) for each task. It may be observed that, although all participants started their tasks at a set distance of $60 \mathrm{~cm}$, viewing distance changed during the 10-minute period. Thus, whereas during the game, text completion and web search tasks a slightly shorter viewing distance was measured $(54.4 \mathrm{~cm}$ [Cl95\% 51.3 to $57.5 \mathrm{~cm}$ ], $54.5 \mathrm{~cm}$ [Cl95\% 51.1 to $58.0 \mathrm{~cm}$ ] and $54.5 \mathrm{~cm}$ [CI95\% 51.4 to $57.7 \mathrm{~cm}$ ], respectively), participants settled at an average of $62.3 \mathrm{~cm}$ [Cl95\% 58.9 to $65.7 \mathrm{~cm}$ ] when tasked with watching a video. When submitted to an ANOVA analysis, a statistically significant difference was found between average distance values as a whole $(F=5.447 ; p=0.002)$. Further pair-wise exploration of this 
difference with a Bonferroni test revealed statistically significant differences between the

240 video task and the other three tasks ( $p$ values of $0.008,0.009$ and 0.010 for the game, text completion and web search tasks, respectively). to $27 \mathrm{~cm}$. Therefore, even if mean viewing distance was close to the initial set value of $60 \mathrm{~cm}$, within each 10 minute evaluation interval participants did not remain stationary at the initial distance, placing themselves alternately at shorter and longer distances while conducting their particular tasks. For instance, distance measurements for one specific participant while performing the video and text completion tasks are plotted in Figure 5. The horizontal line at $40 \mathrm{~cm}$ denotes a minimum recommended viewing distance. It may be observed that this participant had a preferred viewing distance for each task: about $54 \mathrm{~cm}$ for the video task and about $40 \mathrm{~cm}$ while conducting the text completion task. However, during the 10-minute duration of the task the participant kept changing to shorter or longer distances from the display, sometimes going under the threshold of $40 \mathrm{~cm}$. From this information it is possible to measure the range of distances that a given participant uses during the development of each task. It is also noticeable from Figure $\mathbf{5}$ that approximately the first ten measures are more irregular than the other set of measurements. This pattern was common for all tasks and participants, suggesting that during the first minute of each task the participant is deciding on the most comfortable viewing distance.

Finally, upon examining possible associations between the variables under study with the Pearson correlation test, a moderate to strong statistically significant positive correlation was found between the average viewing distances of many of the tasks (Table 2), that is, in general, participants opting to complete one task at a shorter viewing distance also preferred shorter distances for the other tasks. 
264 The main objective of the present study was to develop an affordable (price of each sensor is 265 about $10 \$$ ), non-intrusive method to evaluate viewing distance of VDT users and to test it on a sample of participants undertaking four typical computer tasks. Previous efforts at assessing viewing distance in real time in computer users rely on either infrared ${ }^{19}$ or ultrasound complex systems, ${ }^{27}$ requiring part of the equipment, or at least some markers, to be placed on the forehead of the participants. Therefore, it is believed that these approaches lack operability in that they involve non-trivial installation and configuration, and may interfere with the task being conducted by the participants. The present approach is almost plug-and-play, and no expertise is needed to upload the software to the local computer and to keep the sensor running silently in the background while it monitors viewing distance. In fact, the software also contemplates a remote mode of operation with which a central server computer may govern several sensors installed at different local computers without the need for any further local software configuration. In this regards, it may be easily implemented in a working or academic environment, as well as at home on a personal computer.

It may be noted that in its current configuration the instrument lacks the temporal and spatial resolution of previous devices, with measurements conducted every 7 seconds and a maximum distance resolution of $1 \mathrm{~cm}$. However, within these limitations, the sensor was found to provide repeatable and accurate measurements when a series of consecutive recordings were conducted at controlled prefixed distances of $20,40,60,80$ and $100 \mathrm{~cm}$.

When testing our sensor on a sample of VDT users undertaking different tasks, the equipment revealed the influence of the type of task on viewing distance. In effect, interactive tasks (text completion, web searching and game) were associated with shorter viewing distances than the non-interactive video watching task. However, in disagreement with previous research, ${ }^{19}$ in 
which no details on the study sample are provided, no statistically significant difference was

288 found between the text completion and the web browsing tasks. It may be noted that previous research has documented a relationship between the type of task being conducted by computer operators and aspects such as eyeblink ${ }^{28}$ rate and visual stress or fatigue. ${ }^{29}$ These authors attributed their findings on the actual cognitive demands associated with each task, with more difficult tasks resulting in a reduction in eyeblink rate ${ }^{28}$ and an increase in visual fatigue. ${ }^{29}$ Although the present research investigated similar tasks to those described by these authors, further research is required to determine whether viewing distance is regulated by task difficulty or by other undisclosed factors.

The present findings served to underline that viewing distance may be considered an intrinsic attribute of each individual. In effect, even if viewing distance was found to depend on the type of task, participants were consistent throughout the four different tasks when opting for either short or long viewing distances. Besides, even though all participants conducted all tasks with their habitual correction, and any subjects with binocular vision abnormalities were excluded from the study, it may be speculated whether small differences in such binocular vision function parameters as amplitude and flexibility of accommodation may account for differences in preferred viewing distance. Likewise, given the reported relationship between This first version of the software included a crude feedback mechanism consisting of a small red or green circle appearing on the lower right-hand side of the screen (right side of Figure 5). This notification, which could be set at any distance or deactivated, advised computer users of 
312 their correct or incorrect viewing habits. Further research shall be devoted to design new,

313 more effective feedback strategies, such as switching off the display or progressively reducing

314 its luminance.

315 In conclusion, the present findings revealed a task dependence on viewing distance in 316 computer users. The implications of our results on such relevant issues as myopia onset and

317 progression or visual fatigue require further research. The design and implementation of non-

318 intrusive real-time distance monitoring mechanisms could be the first step towards developing

319 effective feedback strategies to advice computer and other VDT users to maintain correct 320 viewing habits both at home and at during work.

321 
322 ACKNOWLEDGMENTS

323 The authors (GC \& EPC) thank the Spanish Ministerio de Economía y Competitividad and 324 Fondos FEDER for financial support (project number DPI2013-43220-R).

325 
1. http://www.census.gov/hhes/computer/files/2012/Computer Use Infographic FINAL . $\mathrm{pdf}$

2. Hultgren $H$, Knave B. Discomfort glare and disturbances from light reflections in an office landscape with CRT display terminals. Appl Ergon 1974;5:2-8.

3. Thomson WD. Eye problems and visual display terminals -the facts and the fallacies. Ophthalmic Physiol Opt 1998;18:111-9.

4. Cole BL, Maddocks JD, Sharpe K. Effect of VDUs on the eyes: report of a 6-year epidemiological study. Optom Vis Sci 1996;73:512-28.

5. Lie I, Watten RG. VDT work, oculomotor strain, and subjective complaints: an experimental and clinical study. Ergonomics 1994;37:1419-33.

6. Scheiman M. Accommodative and binocular vision disorders associated with video display terminals: diagnosis and management issues. J Am Optom Assoc 1996;67:5319.

7. Rosenfield M. Computer vision syndrome: a review of ocular causes and potential treatments. Ophthal Physiol Opt 2011;31:502-15.

8. Lin YH, Chen CY, Lu SY, Lin YC. Visual fatigue during VDT work: effects of time-based and environment-based conditions. Displays 2008;29:487-92.

9. Hollands JG, Parker HA, McFadden S, Boothby R. LCD versus CRT displays: a comparison of visual search performance for colored symbols. Hum Factors 2002;44:210-21.

10. Sheedy J, Smith R, Hayes J. Visual effects of the luminance surrounding a computer display. Ergonomics 2005;48:1114-28.

11. Shieh KK, Lai YK. Effects of ambient illumination, luminance contrast, and stimulus type on subjective preference of VDT target and background color combinations. Percept Mot Skills 2008;107:236-52. 
12. Shieh KK, Chen MT. Effects of screen color combination, work-break schedule, and workplace on VDT viewing distance. Int J Ind Ergonom 1997;20:11-8.

13. Jaschinski-Kruza W. Visual strain during VDU work: The effect of viewing distance and dark focus. Ergonomics 1988;31:1449-65.

14. Helander MG, Rupp BA. An overview of standards and guidelines for visual display terminals. Appl Ergon 1984;15:205-12.

15. ISO 9241-5, Ergonomic Requirements for Office Work with Visual Display Terminals (VDTs) - Part 5: Workstation Layout and Postural Requirements. Geneva: ISO; 1998.

16. MIL-STD-1472-C, Military Standard, Human Engineering Design Criteria for Military Systems, Equipment and Facilities. Washington DC: US Department of Defense; 1981.

17. DIN 66234, Characteristic Values for the Adaptation of Workstations with Fluorescent Screens to Humans. Berlin: German DIN Association; 1981.

18. Swann M. Ergonomic Recommendations for Work at a VDT Workstation. Surrey, UK: Ergonomic Solutions Limited; 2003.

19. Eastwood-Sutherland C, Gale TJ. Eye-screen distance monitoring for computer use. Conf Proc IEEE Eng Med Biol Soc 2011;2164-7.

20. Sheedy JE, Shaw-McMinn PG. Diagnosing and Treating Computer-related Vision Problems. Burlington, MA: Butterworth Heinemann; 2003.

21. Edwards MH, Lam CS. The epidemiology of myopia in Hong Kong. Ann Acad Med Singapore 2004;33:34-8.

22. Shih YF, Chiang TH, Hsiao CK, Chen CJ, Hung PT, Lin LL. Comparing myopic progression of urban and rural Taiwanese schoolchildren. Jpn J Ophthalmol 2010;54:446-51.

23. Muhamedagic L, Muhamedagic B, Halilovic EA, Halimic JA, Stankovic A, Muracevic B. Relation between near work and myopia progression in student population. Mater Sociomed 2014;26:100-3.

24. Wu LJ, You QS, Duan JL, Luo YX, Liu LJ, Li X, Gao Q, Zhu HP, He Y, Xu L, Jonas JB, Wang $\mathrm{W}$, Guo $\mathrm{XH}$. Prevalence and associated factors of myopia in high-school students in Beijing. PLoS One. 2015; 10: e0120764. 
25. Lee YY, Lo CT, Sheu SJ, Lin JL. What factors are associated with myopia in young adults? A survey study in Taiwan Military Conscripts. Invest Ophthalmol Vis Sci 2013;54:102633.

26. Smith TS, Frick KD, Holden BA, Fricke TR, Naidoo KS. Potential lost productivity resulting from the global burden of uncorrected refractive error. Bull World Health Organ 2009;87:431-7.

27. Piccoli B, D'Orso M, Zambelli PL, Troiano P, Assini R. Observation distance and blinking rate measurement during on-site investigation: new electronic equipment. Ergonomics 2001;44:668-76.

28. Himebaugh NL, Begley CG, Bradley A, Wilkinson JA. Blinking and tear break-up during four visual tasks. Optom Vis Sci 2009;86:E106-14.

29. Gowrisankaran S, Nahar NK, Hayes JR, Sheedy JE. Asthenopia and blink rate under visual and cognitive loads. Optom Vis Sci 2012;89:97-104.

394

395 
397 Figure 1. Beam pattern of the transducer used on the ultrasonic sensor. Receiving sensitivity is $398-65 \mathrm{~dB}$ at $40 \mathrm{KHz}$ according to technical specifications of manufacturer. Sensitivity reduction at 399 various distances is shown in $\mathrm{dB}$.

400

401

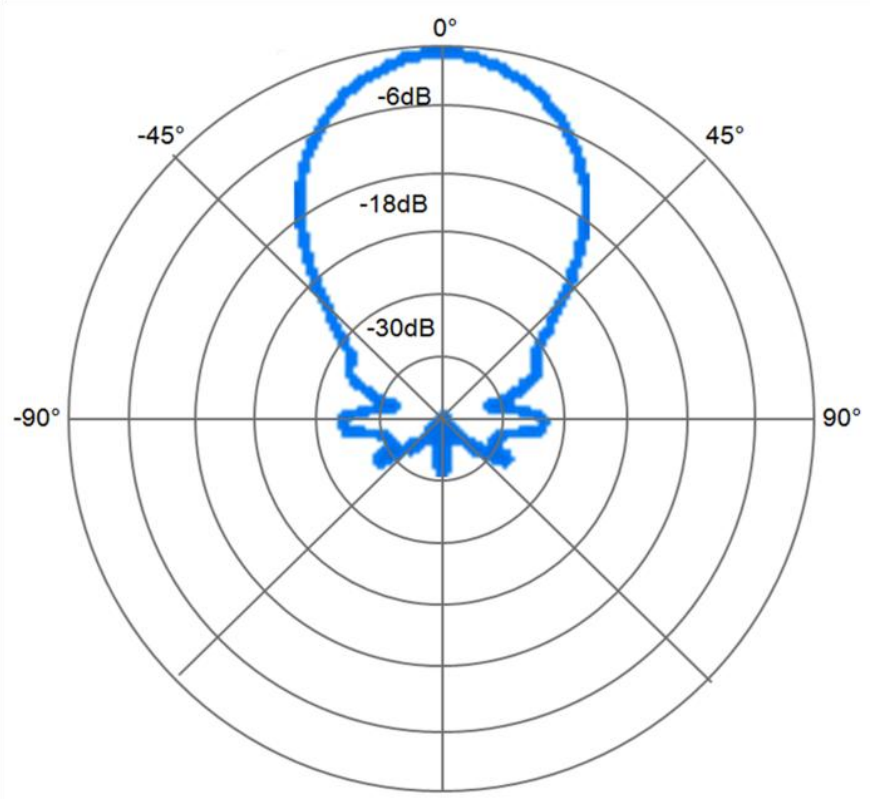


402 Figure 2. Sensor and USB adapter fitted inside a webcam case.

403

404

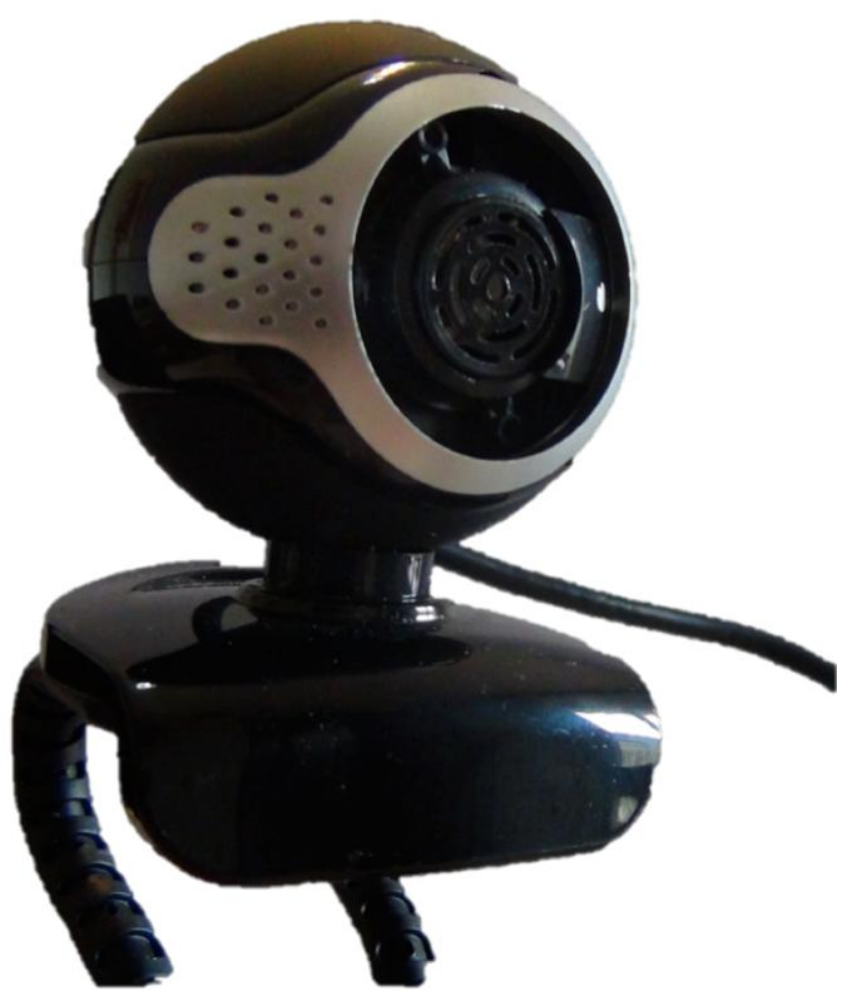

405 

407 horizontal line at $60 \mathrm{~cm}$ denotes initial viewing distance for all tasks.

408

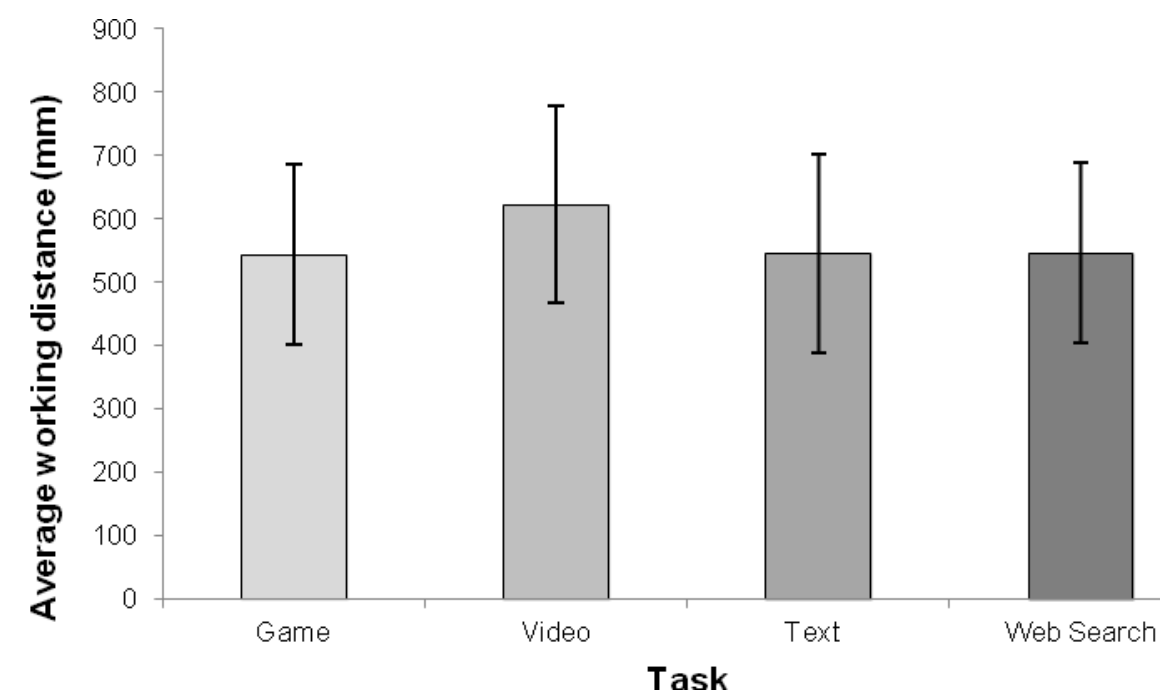

409

410 
411 Figure 4. Average range of distances (in $\mathrm{mm}$ ) for each task ( $\pm 2 \mathrm{SD}$ error bars are shown).

412

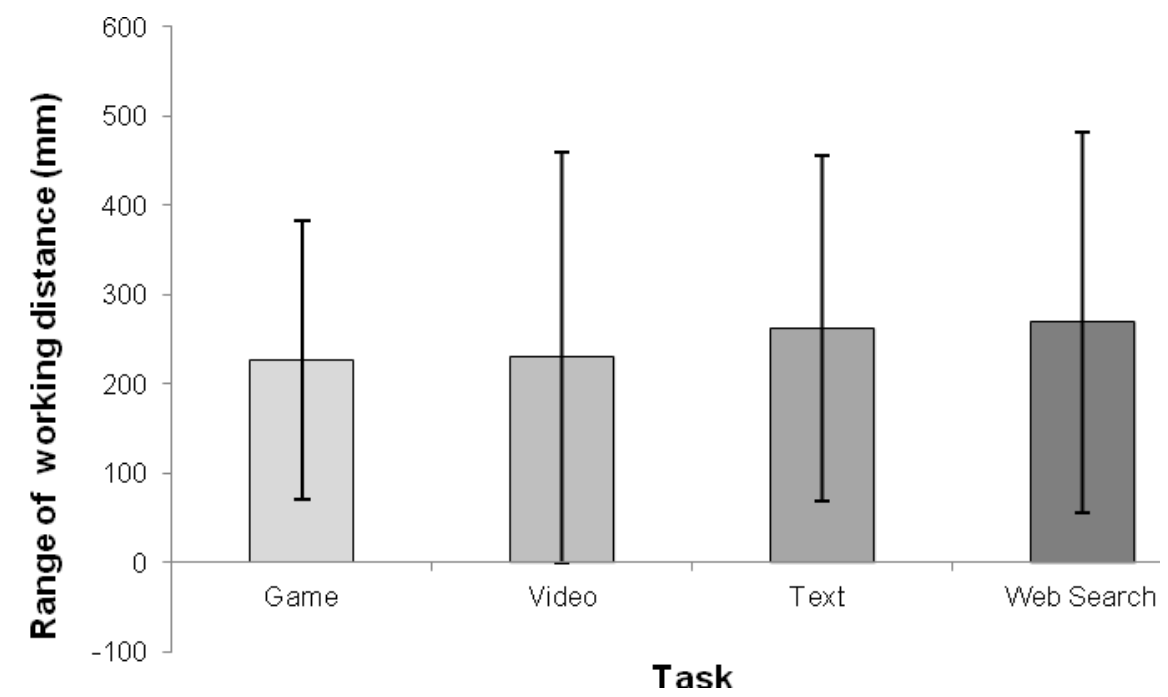

413

414 
Figure 5. Consecutive sensor measurements within a 10 minute interval. Two tasks are drawn 416 (watching a video and completing a text). A $40 \mathrm{~cm}$ reference threshold is shown. The software may be configured to present a red or green on-screen notification as a feedback mechanism when observers are beyond or within the recommended viewing distance, respectively.

419

420

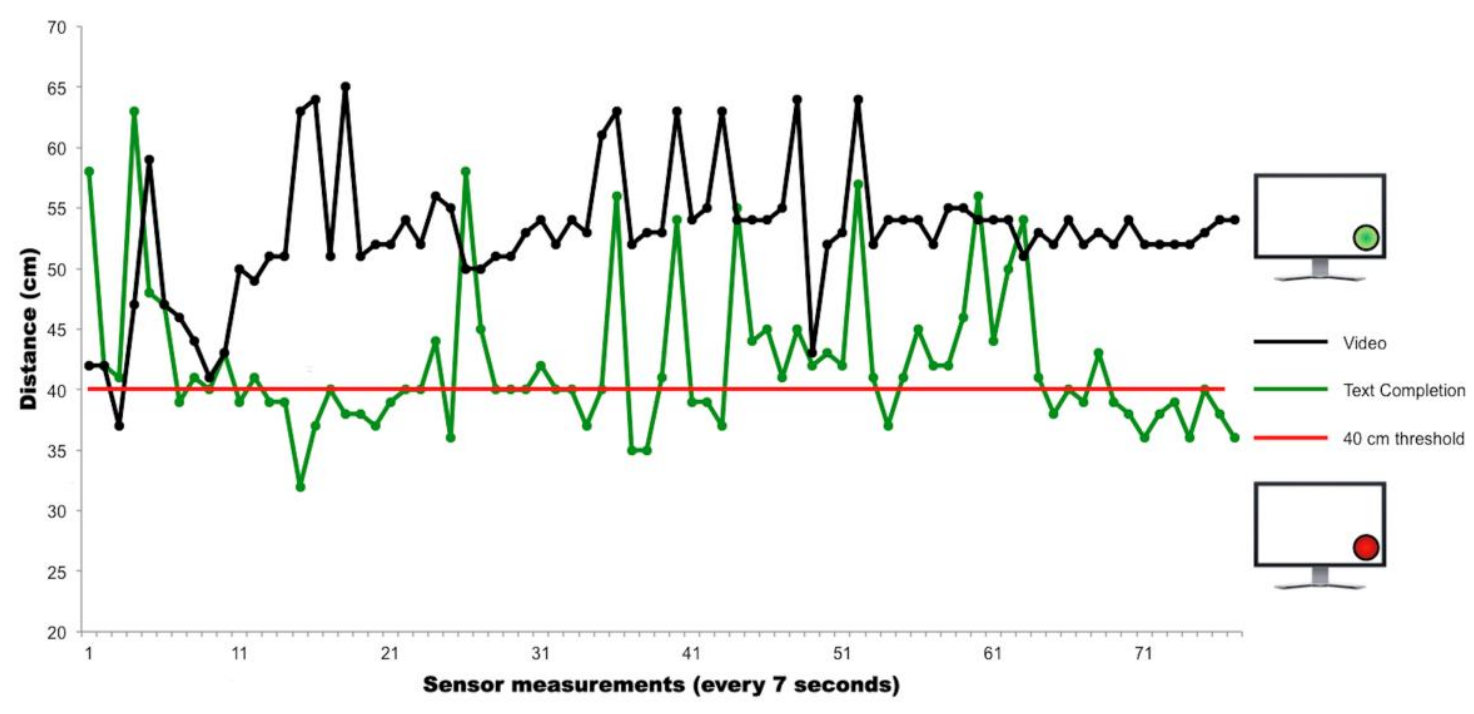


424 Table 1. Average distance and range of observation distances (difference between maximum 425 and minimum observation distance) for each task (game, video, text completion, web search). 426 Data is presented as mean and standard deviation $( \pm S D)$. The longest average working 427 distance corresponded to the video task, with small, not significant differences between the 428 other three tasks. The range of observation distances over the 10-minute interval was similarly 429 large for all tasks.

430

\begin{tabular}{ccc}
\hline TASK & Observation Distance $\mathbf{( c m )}$ & Range of Observation Distances $(\mathbf{c m})$ \\
\hline Game & $54.4 \pm 7.1$ & $22.6 \pm 7.8$ \\
Video & $62.3 \pm 7.8$ & $22.9 \pm 11.5$ \\
Text Completion & $54.5 \pm 7.8$ & $26.2 \pm 9.7$ \\
Web Search & $54.5 \pm 7.1$ & $26.9 \pm 10.7$ \\
\hline
\end{tabular}

431 
434 Table 2. Correlations between mean observation distances for the different tasks. Pearson

435 coefficient of correlation, $r$, and statistical significance, $p$ (between brackets), are presented.

436 The highest correlation was found between the web search and text completion tasks,

437 whereas average working distances for video and game were not correlated. Overall these

438 findings suggest that each subject consistently selects shorter or longer working distances to

439 conduct most tasks.

440

\begin{tabular}{ccccc}
\hline TASK & Game & Video & Text Completion & Web Search \\
& & & & \\
\hline \multirow{2}{*}{ Game } & - & 0.327 & 0.597 & 0.473 \\
& & $(0.159)$ & $(0.005)$ & $(0.035)$ \\
Video & & 0.629 & 0.624 \\
& & & $(0.003)$ & $(0.003)$ \\
Text Completion & & - & 0.740 \\
& & & & $(<0.001)$ \\
\hline
\end{tabular}

441

442 